\title{
Atomic Beam Correlations and the Quantum State of the Micromaser
}

\author{
Per Elmfors* a) ${ }^{*}$ Benny Lautrup $\left.{ }^{\dagger} \quad a, b\right)$, and Bo-Sture Skagerstam ${ }^{\ddagger} a, c$ ) \\ a) CERN, TH-Division, CH-1211 Geneva 23, Switzerland \\ ${ }^{b)}$ CONNECT, The Niels Bohr Institute, Blegdamsvej 17, \\ DK-2100 Copenhagen, Denmark \\ ${ }^{c}$ Institute of Theoretical Physics, Chalmers University of Technology, \\ Göteborg University, S-412 96 Göteborg, Sweden, \\ and Department of Physics, University of Trondheim, N-7055 Dragvoll, Norway
}

\begin{abstract}
Correlation measurements on the states of two-level atoms having passed through a micromaser at different times can be used to infer properties of the quantum state of the radiation field in the cavity. Long(short) correlation length in time is to some extent associated with super(sub)-Poissonian photon statistics. The correlation length is also an indicator of a phase structure much richer than what is revealed by the usual single-time observables, like the atomic inversion or the Mandel quality factor. In realistic experimental situations the correlations may extend over many times the decay time of the cavity. Our assertions are verified by comparing theoretical calculations with a high-precision Monte-Carlo simulation of the micromaser system.
\end{abstract}

The one-photon atomic transition micromaser [1] vides an impressive experimental realization of the interaction between a two-level atom and a second quantized singlemode electromagnetic field. The microlaser [ $[5]$ is the counterpart in the optical regime. Here we focus our interest on the micromaser system for which quantum collapse and signs of quantum revival have been observed [6]. A superconducting niobium cavity, cooled down to a temperature of $T=0.5 \mathrm{~K}$ (corresponding to a thermal photon occupation number of $n_{b}=0.15$ at the maser frequency of $21.5 \mathrm{GHz}$ ), has been used to study the quantum state of the radiation field [7, 8 ]. The high quality factor of the cavity corresponds to a photon lifetime of $T_{\text {cav }}=0.2 \mathrm{~s}$.

In this context, a basic physical feature is the close connection between the cavity's steady-state photon statistics and the fluctuations in the number of atoms in the lower maser level, for a fixed cavity transit time $\tau$ of the atoms [9, 10]. The experimental results of [ [ appearance of non-classical, sub-Poissonian statistics of the radiation field, and exhibit the intricate relation between the atomic beam and the quantum state of the cavity.

In this letter we study in more detail this relation, and discuss the role of correlations for revealing the quantum state of the micromaser system. The sequence of outgoing atoms is viewed as a one-dimensional binary spin chain, each spin representing the state of an atom after the interaction with the cavity. The spin average is closely related to what is usually called the atomic inversion. From the second-order correlation functions of the spin chain we are immediately led to the physical concept of an atomic correlation length $\xi_{A}$, charac-

\footnotetext{
*email: elmfors@cern.ch

†email: lautrup@connect.nbi.dk

‡email: tfebss@fy.chalmers.se
}

terizing the long-range correlations in time in the outgoing atomic beam.

By means of Monte Carlo simulations we study the dynamical generation of such correlations in the atomic beam and how these would show up in an actual experimental situation. We discuss the set of characteristic dynamic phases revealed by $\xi_{A}$, and the implications for the underlying photon distribution in the cavity.

The theory of the micromaser has been developed in [9], and we follow the notation of that paper. In the experiments, excited atoms are injected into the cavity at a rate $R$ high enough to pump up the cavity from vacuum, i.e. $R>\gamma=1 / T_{\text {cav }}$, or $N>1$ in terms of the dimensionless flux variable $N=R / \gamma$. The atom-field interaction time $\tau$ is so much shorter than the average time between two atoms, $\bar{T}=1 / R$, that with a very high probability only one atom at a time is found inside the cavity [11]. Since $\tau$ is also much shorter than $T_{\text {cav }}$, damping effects may be ignored while the atom passes through the cavity. If an atom enters at time $t$ and interacts for a time $\tau$, then the statistical operator of the whole system changes to $\rho(t+\tau)=e^{-i H \tau} \rho(t) e^{i H \tau}$, where $H$ is the total Hamiltonian of the atom-field interaction. It can be approximated by the Jaynes-Cummings (JC) Hamiltonian [12]. This Hamiltonian has the property that it only affects the reduced density operator $\rho_{F}(t)=\operatorname{Tr}_{A}(\rho(t))$ of the radiation field along its diagonals, so that if $\rho_{F}(t)$ is diagonal - which we assume - then so is $\rho_{F}(t+\tau)$. Writing $\rho_{F}(t)=\sum_{n=0}^{\infty} p_{n}(t)|n\rangle\langle n|$ we may express the above interaction as a linear operator acting on the infinite-dimensional vector $p(t)=\left\{p_{0}(t), p_{1}(t), \ldots\right\}$, i.e. as $p(t+\tau)=M(\tau) p(t)$, where $M=M^{+}+M^{-}$is composed of two parts, representing either that the photon occupation number is unchanged (the atom is in state + ), or that it is increased by 1 due to the decay of the atom (the atom is in state -). From the JC-model we have 


$$
\begin{aligned}
& M_{n m}^{+}(\tau)=\left(1-q_{n+1}(\tau)\right) \delta_{n m}, \\
& M_{n m}^{-}(\tau)=q_{n}(\tau) \delta_{n-1, m},
\end{aligned}
$$

where at resonance between the cavity mode and the atomic transition $q_{n}(\tau)=\sin ^{2}(\Omega \tau \sqrt{n})$. The quantity $\Omega$ is the singlephoton Rabi frequency [12].

Let the next atom arrive in the cavity after a time $T \gg \tau$. During this interval the cavity damping is described by a conventional master equation, which also preserves the diagonal form of the cavity density matrix. It may be brought to the form $\dot{p}(t)=-\gamma L p(t)$, where $\gamma$ is the characteristic damping constant of the cavity and $L$ is the matrix:

$$
\begin{aligned}
L_{n m}= & \left(n_{b}+1\right)\left(n \delta_{n, m}-(n+1) \delta_{n+1, m}\right) \\
& +n_{b}\left((n+1) \delta_{n, m}-n \delta_{n-1, m}\right) .
\end{aligned}
$$

The statistical state of the cavity when the next atom arrives is thus given by

$$
p(t+T)=e^{-\gamma L T} M(\tau) p(t) .
$$

The time intervals between atoms are assumed to be Poissondistributed $d \mathcal{P}(T)=\exp (-R T) R d T$ with an average interval $\bar{T}=1 / R$ (for a discussion of non-Poissonian beam statistics see [13], and consequently we may average the exponential of Eq. (3) to get

$$
p(t+\bar{T}) \simeq \frac{1}{1+\gamma \bar{T} L} M(\tau) p(t) \equiv S(\tau, \bar{T}) p(t) .
$$

The equation for statistical equilibrium thus becomes $M(\tau) \bar{p}=(1+\gamma \bar{T} L) \bar{p}$, which has the solution [9.14] for $n \geq 1$

$$
\bar{p}_{n}=\bar{p}_{0} \prod_{m=1}^{n} \frac{n_{b}}{n_{b}+1}\left(1+\frac{q_{m}}{\gamma \bar{T} n_{b} m}\right) .
$$

The overall constant $\bar{p}_{0}$ is determined by $\sum_{n=0}^{\infty} \bar{p}_{n}=1$.

Let the state of an atom emerging from the cavity be characterized by a binary "spin" variable $s= \pm 1$ where +1 denotes the excited level. In statistical equilibrium the probability $\mathcal{P}(s)$ of finding the atom in the state $s$ after the interaction is given by $\mathcal{P}(s)=u^{\top} M^{s} \bar{p}$ where $u$ is a vector with all entries equal to one, representing the trace over the cavity. If the detection efficiency is not $100 \%$ these formulas are modified accordingly [15]. The average spin is $\mu=\langle s\rangle=\mathcal{P}(+)-\mathcal{P}(-)$ (and the atomic inversion $-\mu / 2$ ). Its variance is $\sigma^{2}=\left\langle(s-\mu)^{2}\right\rangle=1-\mu^{2}$. From the spin average we may in statistical equilibrium determine the average occupation number of the cavity mode $\langle\hat{n}\rangle=n_{b}+(1-\mu) / 2 \gamma \bar{T}$.

The joint probability $\mathcal{P}_{k}\left(s_{1}, s_{2}\right)$ for observing the states of two atoms, with $k$ unobserved atoms between them, is given by

$$
\mathcal{P}_{k}\left(s_{1}, s_{2}\right)=u^{\top} M^{s_{2}} S^{k} \frac{1}{1+\gamma \bar{T} L} M^{s_{1}} \bar{p},
$$

where the matrix $S=S(\tau, \bar{T})$ is defined in Eq. (4). From this we derive the $k$-step correlation function $\langle s s\rangle_{k}=\sum_{s_{1}, s_{2}} s_{1} s_{2} \mathcal{P}_{k}\left(s_{1}, s_{2}\right)=\mathcal{P}_{k}(+,+)+\mathcal{P}_{k}(-,-)-$ $\mathcal{P}_{k}(+,-)-\mathcal{P}_{k}(-,+)$. It is easy to show that $\mathcal{P}_{k}(+,-)=$ $\mathcal{P}_{k}(-,+)$ using the stationarity condition for $\bar{p}$. The normalized atomic correlation is $\gamma_{k}^{A}=\left(\langle s s\rangle_{k}-\langle s\rangle^{2}\right) / \sigma^{2}$.

The state of the cavity can be characterized by the average $\langle\hat{n}\rangle$ of the occupation number operator $\hat{n}$ and higherorder observables such as the Mandel quality factor $Q_{f}=$ $\left\langle(\hat{n}-\langle\hat{n}\rangle)^{2}\right\rangle /\langle\hat{n}\rangle-1$, which has the property of being negative for sub-Poissonian (and non-classical) states of the field [16]. We may further characterize the state of the radiation field by correlations between cavity observables at different times. If for example the occupation number $\hat{n}$ is measured twice, with $k$ unobserved atoms passing in between, we consider the correlation function

$$
\langle\hat{n} \hat{n}\rangle_{k}=u^{\top} \hat{n} S^{k} \hat{n} \bar{p}
$$

As before we define the normalized correlation to be $\gamma_{k}^{F}=$ $\left(\langle\hat{n} \hat{n}\rangle_{k}-\langle\hat{n}\rangle^{2}\right) /\left\langle(\hat{n}-\langle\hat{n}\rangle)^{2}\right\rangle$.

If $k$ is sufficiently large we define the asymptotic correlation lengths $\xi_{A}$ and $\xi_{F}$ by

$$
\gamma_{k}^{A, F} \simeq \gamma^{A, F} \exp \left(-\frac{k}{R \xi_{A, F}}\right),
$$

which isolates the $k$-dependence. The $R$-factor secures that the correlation lengths are measured in units of physical time. That $\xi \equiv \xi_{A}=\xi_{F}$ follows from the fact that the time evolution is governed by the same matrix in both Eqs. (6) and (7). The correlation length is therefore a convenient probe of the dynamics of the micromaser system.

The generation of a sequence of outgoing atoms is a Markov process defined by the matrix $S=(1+$ $\gamma \bar{T} L)^{-1} M(\tau)$, and correlations over long times are governed by its eigenvalues $\lambda_{n}$. They can be shown to be real, nondegenerate and bounded by $1=\lambda_{0}>\lambda_{1}>\ldots \geq 0$ [17]. Denoting the corresponding right (left) eigenvectors by $p^{(n)}$ $\left(u^{(n)}\right)$ we can decompose $S^{k}$ as

$$
S^{k}=p^{(0)} \otimes u^{(0)}+\sum_{n=1}^{\infty}\left(\lambda_{n}\right)^{k} p^{(n)} \otimes u^{(n)} .
$$

The contribution to $\gamma_{k}^{A}$ or $\gamma_{k}^{F}$ from the $\lambda_{0}=1$ component of $S^{k}$ cancels out and it is the next-to-leading eigenvalue $\lambda_{1}$ that determines the correlation length through $R \xi=-1 / \ln \lambda_{1}$.

In order to determine $\xi$ we have used two methods, a Monte Carlo simulation of the dynamical process and a direct numerical calculation of $\lambda_{n}$. We consider the maser transition $63 p_{3 / 2} \leftrightarrow 61 d_{5 / 2}$ of ${ }^{85} \mathrm{Rb}$ with the single-photon Rabi frequency $\Omega=44 \mathrm{kHz}$. We choose the rate $R$ to be 50 atoms $/ \mathrm{s}$ in order to be close to the experimental situation of []]. In the 
MC simulation we used a sample of $10^{6}$ atoms for 100 different values of $\tau$ (the corresponding real experiment would take a little less than a month to perform non-stop with $100 \%$ measuring efficiency!). We have compared the MC-data and the theoretical average of the atomic inversion as well as the Mandel quality factor. The agreement between theory and the numerical experiment is excellent and constitutes a consistency check of our computational method. The correlation length is measured by a least-squares fit to the data for $1<k<K$, where $K$ is determined by the onset of noise which almost universally happens when the correlation has fallen to about $0.1 \%$. Typical values of $K$ range from 10 to 250, depending on whether the correlation quickly or slowly drops into the noise. This leads to quite different reliability levels for the extraction of the values of the correlation length. Our results are presented in Fig. 11. The error bars on the MC data represent the precision of the fit to the exponential form Eq. (8), but do not include the systematic errors due to the difficulty in reaching the asymptotic region.

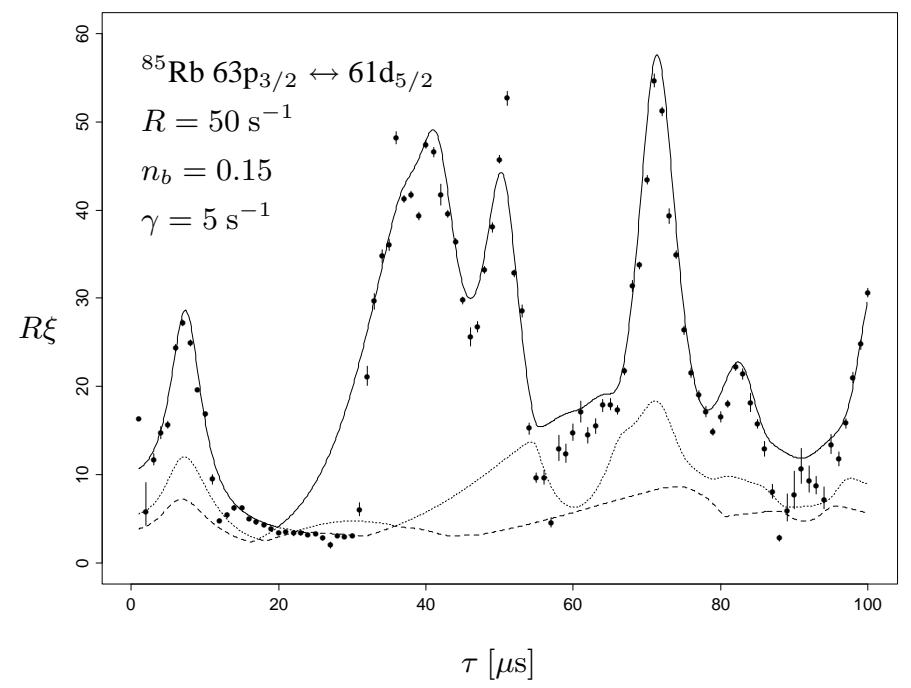

FIG. 1. Comparison of theory (solid curve) and MC data (dots) for the correlation length $R \xi$. The dotted and dashed curves correspond to subleading eigenvalues $\left(\lambda_{2,3}\right)$ of the matrix $S$. The parameters are those of the experiment in [7].

The other numerical method we have used is to find the roots of the equation $\operatorname{det}[M-\lambda(1+\gamma \bar{T} L)]=0$, by truncating the matrix to finite dimension. These roots are the eigenvalues of $S=(1+\gamma \bar{T} L)^{-1} M$. Writing the above equation as an eigenvalue problem for the matrix $M-\alpha L$ with $\alpha=\lambda \gamma \bar{T}$, we recognize that it is a Jacobi matrix (with non-vanishing elements only on the main diagonal and its two neighbouring subdiagonals). The off-diagonal elements are strictly positive for non-negative real $\alpha$. It is known [18] that such a matrix (for fixed $\alpha$ ) has only real and non-degenerate eigenvalues. We have shown that the eigenvalues of $S$ also have these properties, and that $\lambda_{n} \leq 1$, which is required for the convergence of the stochastic process. Numerical calculations of $\lambda_{n}$ converge rapidly for matrices of dimension above 50 . They also agree well with the MC simulation, as can be seen in Fig. 1.
For $\tau \simeq 20-30 \mu$ s the $\mathrm{MC}$ result predicts shorter correlation lengths than the theoretical calculation, but we can see that they agree with a subleading eigenvalue. We surmise that the reason for this is that contributions of the subleading components of $S$ are large for those values of $k$ that can be reached in the MC calculation. In order to extract the actual long-time correlation length in this parameter region, it would be necessary to go much larger $k$ and to increase the statistics considerably. Similar difficulties are expected to be encountered in a real experiment.

The example of the correlation lengths shown in Fig. 1 1have typical parameters of the damping and the flux corresponding to a real experiment [7]. The amount of structure found in this figure is at first glance rather surprising. The mean photon number shows comparatively little structure in this region. We shall now explain the origin of structure in this figure, and refer to Fig. 2, when necessary, which depicts the logarithm of the correlation length for larger flux values. The natural scaling variable in the large flux limit is $\theta=g \tau \sqrt{N}$ which is the one used in Fig. 2. Details of the theoretical derivations will be presented in [17].

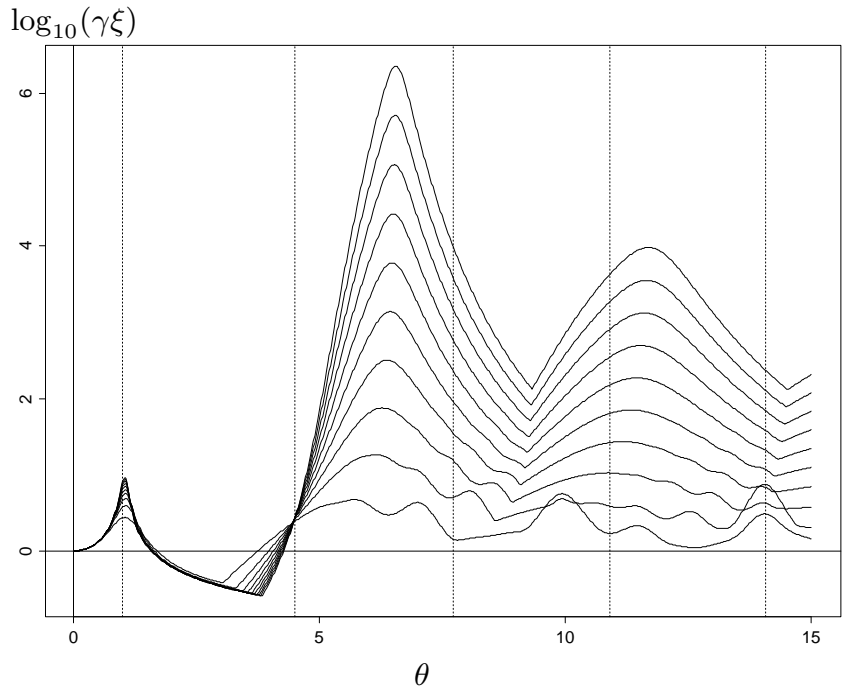

FIG. 2. The logarithm of the correlation length (in units of $1 / \gamma=N / R$ ) for $n_{b}=0.15$ as a function of $\theta=g \tau \sqrt{N}$ for various values of $N=10,20, \ldots, 100$. The vertical lines indicate where new local maxima in the photon distribution function appear. Notice that for $\theta>\theta_{1} \simeq 4.494$, where the photon distribution acquires two maxima, the logarithm of the correlation length grows linearly with $N$ for large $N$.

In the first part of Fig. 1 for $0<\tau<20 \mu s$, the correlation length shows a peak around $\tau=7.2 \mu \mathrm{s}$ which corresponds to the maser transition at $\theta=1$. For smaller values of $\tau$ the field of the cavity has thermal statistics with average number of photons independent of the flux $N(=R / \gamma)$ in the large flux limit. Above the peak, the average photon number grows proportionally with the flux. This peak, which is also clearly present in $\mu$ and $Q_{f}$, is well described by a semiclassical approximation [9,19]. In Fig. 目 we see that in this region the correlation length remains constant in the large flux limit except at the maser transition point where it actually grows like 
$\sqrt{N}$.

At $\tau \simeq 20 \mu \mathrm{s}$, there seems to be crossings of eigenvalues in Fig. 1. Looking closer at these points, we find that there is no actual crossing, consistent with the result mentioned above that there are no degenerate eigenvalues. On the other hand, the corresponding eigenvectors, in this case $p^{(1)}$ and $p^{(2)}$, do cross in the sense that the eigenvector corresponding to $\lambda_{2}$ after the crossing is very close to $p^{(1)}$ before the crossing. This has no analogue in $\mu$ or $Q_{f}$. From the MC simulation we also see that the system may remain dominated by a subleading eigenstate as $\tau$ passes through the transition point. In Fig. we see how this crossing point as the flux increases creeps closer to $\theta_{1} \simeq 4.494$, where the photon distribution function gets a second maximum [17].

Above $\tau \simeq 32 \mu \mathrm{s}$ the correlation length increases dramatically. The reason for that can be traced back to the appearance of two local maxima in the photon distribution function, and the tunneling time between them, as indicated in [9] and calculated explicitly in [17]. We have verified that, as the flux $R$ of atoms increases, the maximal correlation length grows exponentially in this region. For a value of $R=200 / \mathrm{s}$ (which is merely 4 times the flux in the experiment of [7]]), the correlation length at the leading peak extends to 300 times the decay time of the cavity (i.e. a whole minute!). Such a violent behaviour is not reflected in $\mu$ or $Q_{f}$ [9, 19]. For these quantities the most dramatic behaviour is a finite jump in $\mu$ and a narrow peak of finite height in $Q_{f}$ at the second maser transition, which coincides with the exponential peak in $\xi$.

The second maser transition occurs when there is a jump in the position of the global maximum of the photon distribution function. For $N=10$ this happens at $\tau \simeq 51.7 \mu$ s. The three high peaks at $\tau$ around 40, 50 and $70 \mu s$ have, however, a different explanation. They are related to the well-known trapping states [20] which occur when $q_{n}(\tau)$ vanishes. This happens for $\tau \sqrt{n}=k \pi$ with integer $k$. For vanishing $n_{b}$, this effect truncates the equilibrium photon distribution Eq. (5) at a definite photon number. Finite $n_{b}$ smoothes out this effect, but it is still amply visible in Fig. 1. The dominant trapping peaks are numerically given by $\tau=41.2 \mu \mathrm{s}, 50.5 \mu \mathrm{s}, 71.4 \mu \mathrm{s}$ which agrees very well with Fig. 11. In Fig. 2 we see how these peaks are rapidly suppressed relative to the leading and exponentially growing tunneling peak for larger flux. The trapping peaks occur at constant positions in $\tau$ but move away as $\sqrt{N}$ in $\theta$, whereas the tunneling peak remains at fixed $\theta$.

In the semiclassical regime there is a clear connection between the long correlation at the maser transition at $\theta=1$ and the large value of $Q_{f}$, i.e. super-Poissonian photon statistics. For $\theta>\theta_{1} \simeq 4.494$, and for large flux, $Q_{f}$ likewise has peaks at the same positions as $\xi$, namely when there are jumps in the position of the global maximum of the photon distribution. In this sense there is a clear relation between $\xi$ and photon statistics in the cavity. On the other hand, at $\theta_{1}$ for large flux the Mandel factor remains negative while $\xi$ starts growing exponentially, and this growth reveals the appearance of the second local maximum in the photon distribution which is not reflected in the single-time observables.

B.L. and B.-S.S. wish to thank Gabriele Veneziano and the TH-Division for the hospitality at CERN while this work was carried out. The authors also wish to thank I. Lindgren for providing a guide to the experimental work and T.T. Wu for discussions. B.-S. S. acknowledges support by the Swedish National Research Council under contract No. 8244-316, and by the Research Council of Norway under the contract No. 420.95/004.

[1] P. Goy, J.M. Raimond, M. Gross and S. Haroche, Phys. Rev. Lett. 50 (1983) 1903.

[2] D. Meschede, H. Walther and G. Müller, Phys. Rev. Lett. 54 (1985) 551.

[3] H. Walther, Physica Scripta T23 (1988) 165.

[4] H. Walther, in Fundamental Problems in Quantum Theory, Eds. D. M. Greenberger and A. Zeiler, Ann. N.Y. Acad. Sci. 755 (1995) 133.

[5] K. An, J.J. Childs, R.R. Dasari and M.S. Feld, Phys. Rev. Lett. 73 (1994) 3375.

[6] G. Rempe, H. Walther and N. Klein, Phys. Rev. Lett. 58 (1987) 353.

[7] G. Rempe, F. Schmidt-Kaler and H. Walther, Phys. Rev. Lett. 64 (1990) 2783.

[8] G. Rempe, M.O. Scully and H. Walther, Physica Scripta T34 (1991) 5.

[9] D. Filipowicz, J. Javanainen and P. Meystre, Opt. Comm. 58 (1986) 327, and Phys. Rev. A34 (1986) 3077.

[10] G. Rempe and H. Walter, Phys. Rev. A42 (1990) 1650.

[11] E. Wehner, R. Seno, N. Sterpi, B.-G. Englert, and H. Walther, Opt. Comm. 110 (1994) 655.

[12] E.T. Jaynes and F.W. Cummings, Proc. IEEE 51 (1963) 89.

[13] H-J. Briegel and B.-G. Englert, Phys. Rev. A52 (1995) 2361.

[14] L. Lugiato, M. Scully and H. Walther, Phys. Rev. A36 (1987) 740.

[15] H-J. Briegel, B.-G. Englert, N. Sterpi, and H. Walther, Phys. Rev. A49 (1994) 2962.

[16] L. Mandel, Opt. Lett. 4 (1979) 205.

[17] P. Elmfors, B. Lautrup and B.-S. Skagerstam, in preparation.

[18] F.-J. Fritz, B. Huppert and W. Willems, Stochastische Matrizen (Springer-Verlag, 1979).

[19] A.M. Guzman, P. Meystre and E. M. Wright, Phys. Rev. A40 (1989) 2471.

[20] P. Meystre, G. Rempe and H. Walther, Opt. Lett. 13 (1988) 1078. 